\title{
Optimization of Resource Allocation Can Explain the Temporal Dynamics and Honesty of Sexual Signals
}

\author{
Jan Lindström, ${ }^{1, *}$ Thomas W. Pike, ${ }^{1,2}$ Jonathan D. Blount, ${ }^{3}$ and Neil B. Metcalfe ${ }^{1}$ \\ 1. Division of Ecology and Evolutionary Biology, Faculty of Biomedical and Life Sciences, University of Glasgow, Glasgow G12 8QQ, \\ United Kingdom; 2. School of Biology, University of St. Andrews, St. Andrews KY16 9AJ, United Kingdom; 3. Centre for Ecology \\ and Conservation, School of Biosciences, University of Exeter, Cornwall Campus, Penryn, Cornwall TR10 9EZ, United Kingdom \\ Submitted September 30, 2008; Accepted April 7, 2009; Electronically published September 8, 2009
}

ABSTRACT: In species in which males are free to dynamically alter their allocation to sexual signaling over the breeding season, the optimal investment in signaling should depend on both a male's state and the level of competition he faces at any given time. We developed a dynamic optimization model within a game-theoretical framework to explore the resulting signaling dynamics at both individual and population levels and tested two key model predictions with empirical data on three-spined stickleback (Gasterosteus aculeatus) males subjected to dietary manipulation (carotenoid availability): (1) fish in better nutritional condition should be able to maintain their signal for longer over the breeding season, resulting in an increasingly positive correlation between nutritional status and signal (i.e., increasing signal honesty), and (2) female preference for more ornamented males should thus increase over the breeding season. Both predictions were supported by the experimental data. Our model shows how such patterns can emerge from the optimization of resource allocation to signaling in a competitive situation. The key determinants of the honesty and dynamics of sexual signaling are the condition dependency of male survival, the initial frequency distribution of nutritional condition in the male population, and the cost of signaling.

Keywords: game theory, life-history evolution, male-male competition, mate choice, sexual selection.

\section{Introduction}

It is well established that males differ in quality and that females rarely mate randomly, even in species that lack paternal care (Andersson 1994). Females often base their choice on males' secondary sexual characteristics functioning as sexual signals (e.g., Andersson 1994; Hill 2006). These signals can be either relatively static, such as the eye stalk of stalk-eyed flies (Wilkinson and Dodson 1997), or dynamic, as in owl vocalizations (Hardouin et al. 2007). In species in which males are free to dynamically modify

* Corresponding author; e-mail: j.lindstrom@bio.gla.ac.uk.

Am. Nat. 2009. Vol. 174, pp. 515-525. (C) 2009 by The University of Chicago. 0003-0147/2009/17404-50770\$15.00. All rights reserved.

DOI: $10.1086 / 606008$ expression of their sexual signal over the breeding season, it is predicted that the resources a male allocates to signaling will depend on both his own state, as this affects the optimal solution to the expected signaling-survival trade-off (e.g., Mappes et al. 1996; Pike et al. 2007; Judge et al. 2008), and the competition he faces at any given time (Houston and McNamara 1987; McCauley et al. 2000). Consequently, as the optimal signaling level also depends on both the state and the signaling effort of other males in the population, such a situation becomes an evolutionary game. The solution to this game can be viewed as the population's signaling dynamics over the breeding season.

Among the various forms of sexual signals, those based on carotenoid pigments have attracted considerable interest (e.g., Olson and Owens 1998; Alonso-Alvarez et al. 2004; Pérez-Rodríguez 2008). As animals must obtain carotenoids through their diet, and thus their availability may be limiting, allocation of carotenoids to sexual signaling may indicate a male's foraging ability and general quality. In particular, carotenoids have been shown to have antioxidant and immunomodulatory functions, leading to possible trade-offs between sexual signaling and somatic maintenance (Lozano 1994; von Schantz et al. 1999). Indeed, by experimentally manipulating carotenoid availability, several studies have demonstrated that carotenoiddependent sexual signals can convey information about the male's ability to resist parasites, diseases, or oxidative stress (e.g., Blount et al. 2003a; McGraw and Ardia 2003; Kolluru et al. 2006; Pike et al. 2007). Carotenoids can also be used dynamically and strategically, depending on the social context (Candolin 2000b; Gautier et al. 2008).

It is well documented that the degree with which sexual signals indicate some underlying quality or condition (signal honesty) is variable (Johnstone 1995; Candolin 1999, $2000 a$; Sadd et al. 2006) and that, theoretically, perfect honesty is not necessarily expected; it is enough to keep the signaling system in place if the signal is honest "on average” (Johnstone and Grafen 1993; Kokko 1997). How- 
ever, the question of what maintains the variability in signaling strategies, both between and within individuals, has been a rather neglected topic in the sexual-selection literature (Wong and Svensson 2009). To gain a thorough understanding of sexual signaling, it must be considered in a life-history context (Höglund and Sheldon 1998; Badyaev and Qvarnström 2002; Badyaev and Vleck 2007) and in the context of competition between individuals (Kokko 1997). Here, using a combination of gametheoretical modeling and dynamic optimization (Houston and McNamara 1987; McCauley et al. 2000), we explore how a male's nutritional state and the level of competition he faces are expected to affect his signaling dynamics and, consequently, those of the population. While previous models have used state-dependent modeling to study sexual-signaling dynamics, they have not addressed the question of signal honesty and its variation over time. We test key model predictions of signal variability and honesty, using the three-spined stickleback Gasterosteus aculeatus, where female choice is affected by male signaling effort and where males may engage in several breeding bouts over the season (Wootton 1976). However, the model is of broad applicability to other species and life histories and therefore provides more general insights into temporal variation in sexual-signaling dynamics at both the individual and the population level.

\section{Material and Methods \\ Model Description}

We constructed a discrete-time, state-dependent dynamic optimization model, in which a male's behavioral action at any given time depends on his condition, $C$, and the time- and state-dependent behavioral strategy that he adopts. Note that "condition" here can be interpreted as the nutritional state of a male, to keep the model more readily comparable with our experiments. Therefore, for the comparisons between the model output and the experimental data, males in better condition simply have more nutritional resources, such as carotenoids, to deploy for sexual signaling. Following Houston and McNamara (1999), we define a strategy as a state-dependent rule for choosing actions at each time point $t$.

The model is initiated with a distribution of male conditions, using a binomial probability density function as follows. As in the usual binomial probability notation, the parameter $\Psi$ denotes the "probability of success," referring here to the probability of having acquired a nutrient unit. Therefore, the initial mean condition in the population can be varied by changing the parameter $\Psi$, which is bounded to $0-1$; the smaller the value, the poorer the initial mean condition in the population. The expected proportion $\varphi_{i}$ of individuals in a given initial condition $C$, is then given by

$$
\varphi_{i}=f\left(C=i \mid C_{\max }, \Psi\right)=\frac{C_{\max } !}{i !\left(C_{\max }\right) !} \Psi^{i}(1-\Psi)^{C_{\max }-i},
$$

where $i$ is $0,1,2, \ldots, C_{\max }$. This ensures that

$$
\sum_{i=0}^{C_{\max }} \varphi_{i}=1
$$

(e.g., Milton and Arnold 1990). This distribution was adjusted slightly in the model in such a way that at least $1 \%$ of individuals were found in each of the possible starting conditions and the corresponding fraction was evenly subtracted from the other $\varphi_{i}$ values, keeping the overall sum at unity.

At each time step, $t$, individuals in each possible state chose a behavioral action among four alternatives. The first of these did not involve sexual signaling and so represented skipping a reproductive bout and foraging instead. The other actions involved a weak, medium, or strong sexual signal (see table 1 for summary of the actions and their associated nutritional costs). In the case of foraging, the nutrition yield was made stochastic, so that the expected mean nutrition yield, $y$, was a model parameter, with the following outcomes and associated probabilities: $y$, with probability $0.4 ; y+1$ or $y-1$, with probability 0.25 ; and 0 , with probability 0.1 . Males incurred a nutritional cost $H$ at each time step, which was positively correlated with the degree of signaling effort (see table 1). Note that we do not make an explicit distinction here between the cost of signaling per se and the possible associated cost of male-male competition, so one can also consider $H$ as a summary measure of both the cost of signaling and that of male-male competition. The individuals chose among the options available by maximizing the expected lifetime reproductive output given their current state, $R\left(C_{t}, T\right)$, from time $t$ onward, using the dynamic programming equation:

$$
R\left(C_{t}, T\right)=\max \left[M_{i}+s_{i} R\left(x_{i}^{\prime}, t+1, T\right)\right],
$$

Table 1: Nutritional costs of each male action

\begin{tabular}{lcc}
\hline Action & Cost & Signal strength \\
\hline Foraging & 1 & 0 \\
Weak signal & $H-2$ & 1 \\
Medium signal & $H-1$ & 2 \\
Strong signal & $H$ & 3 \\
\hline
\end{tabular}

Note: Foraging is the only action increasing energy, determined by parameter $y$ (see text for details). For survival and mating success, see eqq. (3), (7). 
where $C_{t}$ is condition at time $t ; M_{i}$ and $s_{i}$ are the current mating success and survival, respectively; and $R\left(x_{i}^{\prime}, t+\right.$ $1, T)$ is the remaining reproductive output from time $t+1$ to $T$, assuming that the best condition-dependent action $x_{i}^{\prime}$ is always chosen. "Current" here refers to the expected mating success and survival until the next time step as a result of the chosen level of sexual signaling and reproductive effort. To allow investigation of different scenarios of how survival might affect signaling dynamics and resource allocation, it was set to be exponentially dependent on condition, $C_{t}$ :

$$
s_{i}=k_{1}\left[1-\exp \left(k_{2} C_{t}\right)\right] .
$$

Here, $k_{1}$ sets the asymptotic survival, and $k_{2}$ indicates how fast this is achieved as a function of condition (the more negative $k_{2}$ is, the lower the condition needed for maximum survival is).

To take into account the fact that animal decision making is unlikely to be perfect and to aid convergence of the strategies, we allowed error in decision making $(\mathrm{McNa}-$ mara et al. 1997). The probability of choosing a suboptimal behavioral action was set to be dependent on the cost of the error in terms of lost expected reproductive value, $l_{x(t)}$ :

$$
l_{x(t)}=R\left(C_{t}, T \mid x_{t}^{\prime}\right)-R\left(C_{t}, T \mid x_{t}\right),
$$

where $l_{x(t)}>0$ when an action not maximizing the reproductive value $R_{x_{t}^{\prime}}$ is chosen. To control the amount of error associated with the costs of different choices, we write

$$
\beta_{x(t)}=\exp \left(\frac{-l_{x(t)}}{\delta}\right)
$$

where $\delta$ is the error parameter (if $\delta=0$, there are no errors, and $\delta=1$ corresponds to $\sim 0.5$ probability of making a wrong choice; see, e.g., Houston and McNamara 1999; $\delta$ is set to 0.9 in the results presented here). The $\beta$ values, which are a measure of the potential loss of reproductive value from choosing a given action, weight the probability of that action being chosen at time $t, \operatorname{Pr} x(t)$, as follows:

$$
\operatorname{Pr} x(t)=\frac{\beta_{x(t)}}{\sum_{x=1}^{\Omega} \beta_{x(t)}},
$$

where $\Omega$ is the number of possible actions (here, 4 ). The best action to take at any given time $t$ is defined by these probabilities, instead of simply choosing the one with highest $R_{x(t)}$.

Mating success of a given individual $i$ in condition $C$ at time $t, M_{C, I, t}$, was based on the action-specific signal strength (table 1), weighted by the probabilities of taking a given action (eq. [6]), in relation to other males' signals at time $t$. First, the distribution of signal strengths in the male population at each time step was calculated on the basis of the condition-dependent actions, their associated probabilities (eq. [6]), and the expected proportion of males in a given condition at that time (eq. [8]), assuming a large starting population. The males in the population were then ranked according to their signal strength. The expected number of matings was allowed to range between 0 and 3 , and their associated probabilities were calculated using a binomial probability density function. Mating success probability is written as $\Psi_{M}^{\lambda}$, where $\lambda$ denotes the strength of mating skew, that is, how fast $\Psi_{M}$ changes with signal rank (which ranges from 1 to the total number of males). When $\lambda>0, \Psi_{M}$ decreases with signal rank; large values of $\lambda$ correspond to situations in which only males with the strongest signals are successful in mating. The male with the highest signal rank was always given three matings $\left(i_{\max }=3\right)$, and the probabilities for a male with signal rank $j$ to acquire $i(0,1,2$, or 3$)$ matings, $\omega_{i}$, are

$$
\omega_{i}=f\left(i \mid i_{\max }, \Psi_{M, j}^{\lambda}\right)=\frac{i_{\max } !}{i !\left(i_{\max }\right) !} \Psi_{M, j}^{\lambda}\left(1-\Psi_{M, j}^{\lambda}\right)^{i_{\max }-i},
$$

and his expected total number of matings, $\Omega_{j}$, becomes

$$
\Omega_{j}=\sum_{i=0}^{i_{\max }} \omega_{i} i
$$

The initial state distribution of the males (eq. [1a]) was left to run until $99.9 \%$ of the male population was dead, so the end point of the model, $T$, was not fixed but depended on the model parameters. A state distribution vector $\mathbf{s}(t)$ from the initiation onward was calculated as (e.g., Caswell 2001)

$$
\mathbf{s}(t)=\mathbf{A}(t) \mathbf{s}(t)
$$

where the transition probabilities in the matrix $\mathbf{A}$ were derived using the action-specific parameter values (table 1) and the probabilities of an individual at each state choosing a given action, $\operatorname{Pr} x(t)$ (eq. [6]). Death, $\mathbf{s}(1,[0, T])$, was an absorbing state.

The evolutionarily stable strategy (ESS) was found by changing the time- and state-dependent probabilities of taking an action, $\operatorname{Pr} x(t)$, after each dynamic optimization round to the new optimum and finding the population state distribution for each $t$ with equation (8). The dynamic optimization procedure was then repeated (backward) until the new optimum values of $\operatorname{Pr} x(t)$ were found. 
In most cases, these probabilities converged in 10-20 iterations in the backward optimization of equation (2).

\section{Experimental Design}

Juvenile three-spined sticklebacks were captured with dip nets from the River Endrick, Scotland $\left(56^{\circ} 4^{\prime} \mathrm{N}, 4^{\circ} 23^{\prime} \mathrm{W}\right)$, during November 2005. After capture, individual fish were allocated randomly to one of 12 holding aquariums, each containing only a water filter and several artificial plants (to provide refuges and reduce stress), and held until the start of the breeding season under a simulated natural temperature and photoperiod regime. Throughout the experiment, fish were fed to satiation daily on a customized diet based on anchovy-meal-based fish feed pellets (which are naturally low in carotenoids), supplemented with equal quantities of astaxanthin (Carophyll Pink; DSM, Basel, Switzerland) and lutein (FloraGLO; Kemin Health, Des Moines, IA; which also contains approximately $4.2 \%$ zeaxanthin) at a total concentration of either $200 \mathrm{mg}$ carotenoids $\mathrm{kg}^{-1}$ pellets (high-carotenoid diet) or $10 \mathrm{mg} \mathrm{kg} \mathrm{kg}^{-1}$ (low-carotenoid diet). Full details of diet preparation are given elsewhere (Pike et al. 2007). This concentration and composition of carotenoids is known to allow male sticklebacks to develop normal sexual coloration and successfully engage in reproductive activities. However, while the low-carotenoid diet is sufficient to produce sexual coloration, it does not permit them to maintain this level of signal for as long as does the high-carotenoid diet (Pike et al. 2007). Algal growth was suppressed using 2-chloro4, 6-bis-(ethylamino)-s-triazine (Algae Destroyer; Aquarium Pharmaceuticals, Chalfont, PA), and so we were confident that the only source of carotenoids available to these fish was through the diet.

When males began to develop blue eye coloration (an indicator of sexual maturation), they were transferred to individual experimental aquariums $(33 \mathrm{~cm} \times 18 \mathrm{~cm} \times 19$ $\mathrm{cm})$, where they were maintained on the same experimental diets. The experimental aquariums contained only a filter and an artificial plant and had the same photoperiod, temperature, and water conditions as the holding aquariums. Individual aquariums were separated by opaque partitions, so males were not in visual or olfactory contact with one another. Each male was provided with a nesting dish and nesting material (following Pike et al. 2007) and shown a gravid female enclosed in a clear acrylic plastic container for 5 min twice daily for 10 days, after which males had developed red nuptial coloration and entered the courtship phase. This protocol allowed males to assess the adequacy of their signaling through the females' behavior without the problems associated with dominance interactions between males in artificially close proximity. Pilot experiments showed that a dominance hierarchy would be otherwise quickly established, and the subdominant male would stop signaling and normal courtship behavior, a situation that would not occur in the wild as the territories can be farther apart. We randomly selected 40 males for this experiment, with equal numbers from each diet treatment.

When nest building was complete, a gravid female was placed temporarily in each male's tank and allowed to spawn. The males were then allowed to care for the eggs until the end of the incubation period, when the nests and nesting dishes were removed. After this first breeding attempt, males were induced to build another nest as before and were presented with a new female every 20 days, so that the cycle of breeding continued (exactly as described above) for a maximum of three breeding rounds. Every 20 days, corresponding to the start of each new breeding cycle, we quantified each male's attractiveness to females and the strength of their sexual signal. Attractiveness was assessed by dividing males randomly into pairs so that each high-carotenoid-diet male was paired with an approximately size-matched male of the opposite diet treatment (in no breeding round did pairs of males differ significantly in standard length; paired $t$-tests, all $P>.39$ ). The same pairs were maintained throughout subsequent breeding rounds, and so if a male failed to build a nest, then no trial was conducted for that pair. The trials were conducted following a well-established technique (described in Pike et al. 2007), in which a single gravid female was given a 5-min binary choice between the two males and her preference was quantified as the time spent with the high-carotenoid male as a proportion of the time spent with both males. A score of 0.5 therefore represents no preference for either male, while scores $>0.5$ indicate a preference for the high-carotenoid male. Immediately following mate-choice trials, each male was shown a novel gravid female for $10 \mathrm{~min}$, immediately netted, and photographed using a standardized technique to determine a "redness" score (measured as the proportion of color attributable to the red region of the spectrum; Pike et al. 2007).

\section{Statistical Analysis}

The proportion of males successfully building nests decreased over the season, so that sample sizes differ between breeding rounds and the number of rounds completed differs between males. To account for this, seasonal changes in redness scores were analyzed using linear mixed-effects models, with diet treatment (high or low carotenoid) as a fixed factor and an individual fish's signaling effort over the season as a repeated measure. Subsequent pairwise comparisons were made using paired $t$ tests. Female preference for the redder male was analyzed with a generalized linear mixed-effects model, in which 
the male pair identity was fitted as a random factor, the difference in the redness of the males of each of these pairs in each breeding round as a covariate, and the breeding round as a fixed effect. These analyses were carried out in R 2.5.0 (R Development Core Team 2004).

\section{Results}

The Model

We ran the model with a wide range of parameter values, although figure 1 illustrates its general dynamics under two different survival scenarios (fig. $1 A$ ), depicting the males' signaling trajectories (fig. $1 B$ ) and signal honesty (fig. 1C) with two different initial conditions $(100 \%$ and $50 \%$ of maximum condition). The expected survival and its condition dependency play a strong role in determining the expected signaling dynamics and the honesty of the signal. If the maximum condition-dependent survival probability is relatively high (fig. $1 \mathrm{~A}$, solid line), the strongest sexual signals at the start of the breeding season are often not produced by the individuals starting in the highest condition (see also fig. 2), so that signal strength is not a reliable indicator of condition at this time (i.e., it is dishonest). This is in contrast to a situation in which the overall survival probability is relatively low (fig. $1 \mathrm{~A}$, dotted line; fig. 3); saving resources does not pay back then, leading to males in good condition being more likely to signal this from the start of the season. In other words, if longerterm survival has the potential to contribute to the overall fitness return strongly enough, the males in the best condition out-survive rather than out-signal the weaker competitors, particularly in the beginning of the breeding season. Figure 2 shows the signaling dynamics (fig. $2 A, 2 B$, $2 E, 2 F$ ), mating success (cumulative matings; fig. $2 C, 2 G$ ), and mortality (fig. $2 \mathrm{D}, 2 \mathrm{H}$ ) for all the initial conditions over the breeding season. The panels on the left- and righthand side depict the results for the situation in which survival is less strongly (fig. $2 A-2 D$ ) or more strongly (fig. $2 E-2 H$ ) dependent on condition (fig. $1 A$ ), respectively. The overall signal strength seen across the population is predicted to be somewhat higher (fig. $2 A, 2 B$ ) when the overall survival probability is lower, and there is a clear contrast in how honestly the signal strength reveals the true underlying condition depending on the survival pattern (as revealed by the relationship between initial condition and signal rank; fig. $2 B, 2 F$ ). When the maximum survival probability is higher, males in poorer-than-average condition start the breeding season with relatively high mating success (as a result of signaling strongly), but their early death means that males in better initial condition have a higher mating success over the season as a whole because of their greater number of breeding attempts (fig.


Figure 1: Predicted signaling dynamics through the breeding season with two different survival patterns. A, Scenario 1 (dotted line; $k_{1}=0.5$, $k_{2}=-0.5$ ) and scenario 2 (solid line; $k_{1}=0.9, k_{2}=-0.1$; see eq. [3]). All the other parameter values are identical: initial mean population condition parameter $\Psi=0.25$ (eq. [1a]), highest energy cost $H=4$, energy yield $y=3$, and mating skew $\lambda=0.8$ (eqq. [7]). $B$, Expected signal strength against time for individuals starting with the highest (circles) condition (20) or at half of this (10; triangles). Open dots and dotted lines depict survival scenario 1 , and scenario 2 is illustrated with filled dots and solid lines. $C$, Honesty of the sexual signal in the male population under the two survival scenarios (correlation coefficient between current condition and signal rank; this is undefined for $t=7$ as there is no variation left in signal strength or signal rank at that time; dots and lines as in $B)$.

$2 C, 2 G)$. Typically, a large fraction of the male population dies soon after the initial breeding attempts, and individuals starting in the best condition survive longest (fig. $2 D$, $2 H)$. 



$(\mathrm{E})$


Figure 2: Results summary for all the individual starting conditions over time, using the same parameter values as figure 1 . $A-D$ correspond to survival scenario 1, and $E-H$ show the outcome for survival scenario 2. $A$, E, Signal strength; $B, F$, signal rank. Note that for illustrative purposes we compare the ranks of only 20 individuals, with starting conditions $1-20$, and have reversed the $Z$-axis scale. $C$, $G$, Cumulative expected matings; $D, H$, mortality. 




Figure 3: Timing $(t)$ of peak honesty $(A)$ and maximum life span $(B)$ as a function of the survival parameters $k_{1}$ and $k_{2}$. Other parameters as in figures 1,2 .

The timing of peak honesty in the population (fig. $3 A$ ), as well as maximum life span (fig. $3 B$ ), is strongly dependent on the survival function. The parameter $k_{2}$ here indicates how quickly survival increases toward the asymptotic survival (set by $k_{1}$ ) as a function of condition (eq. [3]). Therefore, increasing values of $k_{2}$ indicate increasing condition dependency in survival in our model (and correspondingly, decreasing values indicate higher condition independency of survival that could be due to predation, for instance). The higher the condition-dependent maximum survival $\left(k_{1}\right)$ is, the later in the breeding season the maximum level of signal honesty occurs. This pattern is further modified by the strength of condition dependency in survival probability; for a given level of maximum survival, peak signaling honesty is generally shown earlier when survival is more strongly condition dependent (fig. $3 A$ ). Maximum life span in the population is achieved when the maximum survival is highest and survival is strongly condition dependent (fig. 3B). This is also the situation in which males in high condition are most likely to allocate less resources to signaling earlier in the season and enjoy the fitness returns of the increased number of (subsequent) mating opportunities instead.

Apart from the survival pattern, the maximum cost $H$ associated with the behavioral actions turned out to be the one with most influence on the ESS outcome, together with the initial mean condition of the male population (fig. 4). The strength of the maximum signal attained over the whole breeding season decreases with increasing signaling cost, whereas the initial mean condition of the males affects this pattern only if signaling is relatively costly (fig. $4 A$ ). The initial mean condition of the males changes the timing of maximum signaling observed in the population; a higher mean condition leads to maximal signaling occurring earlier in the season (fig. $4 B$ ), whereas the cost of signaling has less impact on this pattern. Honesty of the signal peaks earlier in the breeding season if signaling is costly, and the initial mean condition of the males has virtually no effect on this pattern (fig. 4C). The cost of signaling has a clear effect on the predicted average life span in the population, and this effect is particularly strong when the males are in relatively poor initial condition (fig. $4 D)$.

Two additional points are worth considering. At present, the model implicitly assumes that female availability stays constant over the whole breeding season. However, relaxing this assumption by simulating either increasing or decreasing mating opportunities with the progressing season (by letting the mating skew parameter $\lambda$, eqq. [7], change with the breeding season) had little effect on the outcome with any parameter values. Similarly, reducing the relative value of offspring (by reducing their survival probability) born late in the breeding season (e.g., because the food availability or growth period is reduced) did not affect the pattern of signaling by males or its consequences in any significant way. There are two main reasons for these results. First, particularly for males in relatively poor condition, the future is not a very important determinant for choosing an optimum strategy in the beginning of the breeding season as they are very unlikely to survive that long (fig. $2 \mathrm{D}, 2 \mathrm{H}$ ). Second, for males in better condition, the importance of the overall survival pattern and the maximum cost $H$ associated with the behavioral strategies chosen overrules the more subtle implications of changes in offspring value or mating skew-as the males compete for their share of matings, the absolute benefit of this share is a less important factor in determining behavioral strategies than surviving up until that time. However, outsurviving competitors would obviously not pay off in extreme cases in which the future promises very little in terms of further matings; this situation is analogous to low survival, which promotes high investment and honesty in signaling in the beginning of the breeding season (fig. 2).

\section{The Experiment}

Allocation to sexual signaling, as defined by male redness, decreased significantly over successive breeding rounds 

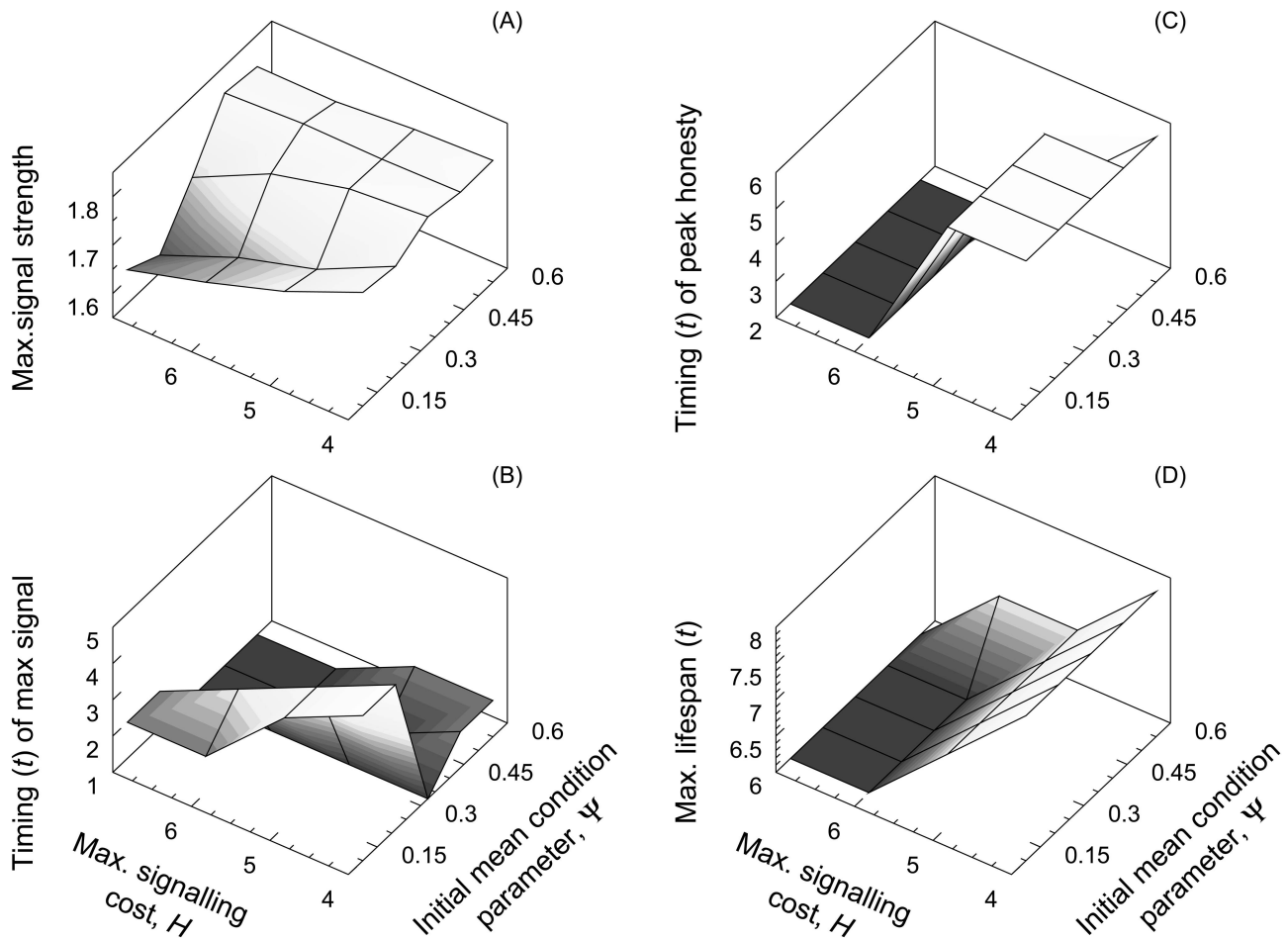

Figure 4: Results summary as a function of two important control parameters, the maximum energy cost of signaling, $H$, and the initial mean starting condition, $\Psi$, of the males (eq. [1a]; the range of $\Psi$ from 0.1 to 0.55 corresponds to the change in the mean initial condition from 4.4 to 11.25). Strength $(A)$ and timing $(B)$ of maximum signal over all the individuals and breeding bouts. $C$, Timing of peak honesty of the signal; $D$, maximum life span (equals maximum expected number of breeding bouts) among the males. Parameters match those of survival scenario 2 in figures 1,2 .

$\left(F_{2,42}=4.16, P=.022\right.$; fig. $\left.5 A\right)$. However, it also differed between diet treatments $\left(F_{1,38}=27.95, P<.001\right)$, and there was a significant interaction between breeding round and $\operatorname{diet}\left(F_{2,42}=9.92, P<.001\right)$. Post hoc analyses revealed that while signaling did not differ between diet treatments in the first breeding round $\left(t_{38}=0.26, P=.797\right)$, the disparity between the groups increased during the second $\left(t_{29}=1.85, P=.075\right)$ and third $\left(t_{13}=6.98, P<.001\right)$ rounds because of a significant reduction in redness of low-carotenoid males but a slight increase in the redness of males on the high-carotenoid diet. Note that the mean signaling level remained fairly constant over all three breeding rounds, despite the marked difference between diet treatment groups.

Female preference for the redder male increased toward the end of the breeding season $\left(F_{2,18}=6.26, P=.009\right.$; fig. $5 B$ ), even though there was no significant difference between the first and the second breeding rounds $\left(t_{32}=\right.$ $1.60, P=.119)$. Male redness affected female preference significantly $\left(F_{1,18}=5.19, P=.035\right)$. The interaction term between breeding round and male redness was not sig- nificant $\left(F_{2,16}=0.47, P=.63\right)$ and was therefore not included in the minimum adequate model.

\section{Discussion}

We set out to explore whether we could explain the diverse patterns observed in the temporal dynamics of sexual signaling and signal honesty within and between individuals by using a combination of a state-dependent dynamic optimization model in a game-theoretical setting and experiments on the three-spined stickleback. Our main findings were that the expected survival and its condition dependency are important determinants for the temporal dynamics of signal honesty. In brief, high overall survival, particularly when condition dependent, can lead to signal dishonesty in the beginning of the breeding season as it is suboptimal for males in good condition to sacrifice their survival by signaling strongly enough to outcompete the males in poorer condition. Such a situation does not occur when the expected survival is low overall since all males are predicted to signal according to their condition from 

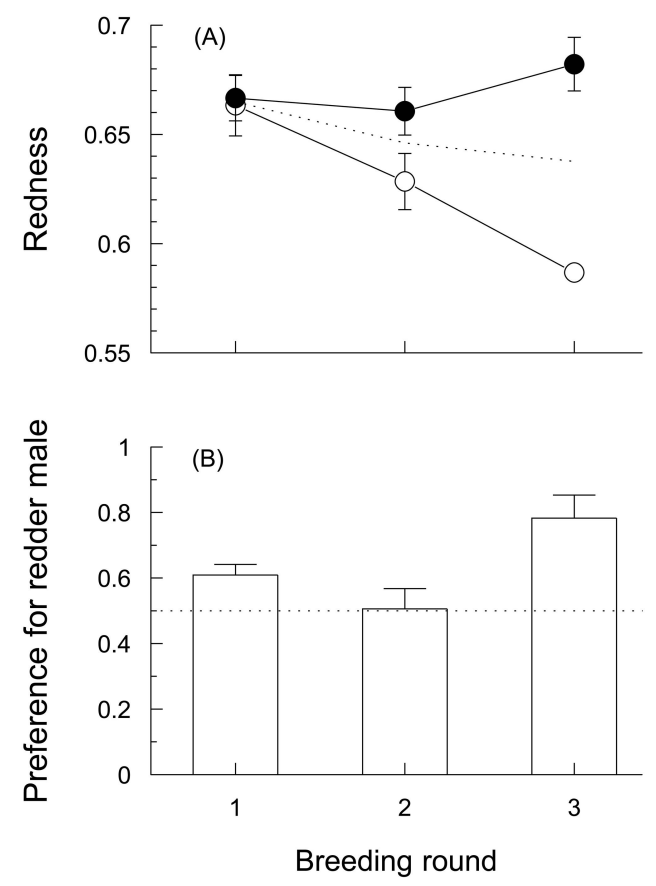

Figure 5: $A$, Mean \pm SE change in redness over successive breeding rounds in males on high-carotenoid diets (filled data points, solid line) and low-carotenoid diets (open data points, dashed line; note that SE of the last data point is too narrow to show behind the marker). The dotted line shows the overall mean redness in different breeding rounds. The number of high- and low-carotenoid-diet males breeding in each round was 20 and 20 (round 1), 17 and 14 (round 2), and 8 and 7 (round 3), respectively. $B$, Mean \pm SE female preference for the redder male in the three breeding rounds (the proportion of time spent with the redder male, where a score of 0.5 represents no preference).

the beginning of the breeding season. Thus, males in good condition are predicted to either out-survive or out-signal the poorer competitors, depending on their survival prospects. The expected signaling pattern is further modified by the cost of signaling and by the initial frequency distribution of male condition in the population. Generally, signal honesty peaks later if the signaling cost is low, which is also the scenario in which individuals are predicted to survive the longest. The strongest effect of mean initial condition of the males is on the timing of peak signaling; increasing average condition brings the signaling competition forward in the season.

In general, patterns predicted by our optimization model when assuming a relatively high and conditiondependent survival rate matched the experimental results well. Individuals from high- and low-carotenoid-diet treatments initially signaled with approximately equal intensity. However, only the fish from the high-carotenoid treatment were able to sustain a relatively high level of signaling over successive breeding rounds. This suggests that the reli- ability of the signal improves with time; males in good nutritional condition should restrict their signaling in the beginning of their breeding career since they have a high chance of outliving males in poorer condition, but high early investment in signaling would reduce their chance for future breeding opportunities (e.g., Candolin 2000a; Proulx et al. 2002; Sparkes et al. 2008; see, however, Hunt et al. 2004). A similar relationship is predicted by the model: the correlation between the signal rank of males and their real underlying condition (i.e., the honesty of the signal) is expected to increase over successive breeding rounds.

Concerning the likelihood of dishonest signaling in natural settings in general, we have shown here that the survival probability and its condition dependency are important factors in determining honesty. In the light of this result, we would expect to see dishonesty in signaling most commonly in mating systems in which the male survival level is reasonably high and condition dependent, in which males can change their signaling level over time, and in which they can have more than one mating opportunity. These conditions are likely to be met across a range of species, including sticklebacks (Candolin 1999, 2000a), crickets Gryllus pennsylvanicus (Judge et al. 2008), and wolf spiders Hygrolycosa rubrofasciata (Mappes et al. 1996). In contrast, situations in which mating is mostly limited to a single opportunity per season should promote honesty in signaling (e.g., foot color in the blue-footed booby Sula nebouxii; Torres and Velando 2003). Similarly, in cases in which survival probability from one breeding attempt to the next is low or the species is semelparous, we predict increased honesty in signaling (see also Proulx et al. 2002). However, we are not aware of any comparative studies contrasting signal honesty or condition dependency in iteroparous versus semelparous species or populations. Our model suggests how such patterns can emerge from the optimization of resource allocation to signaling in a competitive situation.

It has been the prevailing view in the sexual-selection literature that female preferences for male traits remain constant. However, it is now recognized that benefits from mate choice can depend on the genetic match between males and females or the ecological and social context (e.g., Qvarnström 2001; Welch 2003; Suk and Choe 2008). Recently, Chaine and Lyon (2008) showed that individual female lark buntings (Calamospiza melanocorys) change their preferences for particular male traits between years. Also, if a signal is less honest in the beginning of the breeding season, as predicted by our model, when survival is relatively high and condition dependent, it is logical to predict that female preferences based on that signal might also be reduced. Our experimental results lend some support for this prediction (fig. $5 B$ ): females showed a stronger preference for redder males toward the end of the breeding 
season. This opens up an avenue for future research as it would be interesting to know whether this preference is based on increased female choosiness or on increasing differences in signal expression between males. In general, whether females become choosier toward the end of the breeding season is also expected to depend on the potentially changing costs and benefits associated with mate choice. For instance, increasing predation pressure might select against increased choosiness over the breeding season.

It is interesting to note that the overall mean signaling level over the experimental groups remained relatively similar throughout the breeding rounds; this is exactly what the model predicts too. The relatively stable mean signaling effort over the breeding season therefore masks much of the underlying signaling dynamics; the overall signal strength remains seemingly unchanged, but it is different individuals with different strategies and initial conditions that are signaling the most at any given time. As the males received their respective diet treatments throughout the experiment, it is possible that the increase in signal redness over time in high-carotenoid-diet males may have been directly influenced by an increasing availability of carotenoids in the body, whereas the decrease in signal redness over time in low-carotenoid-diet males could have been directly affected by carotenoid levels depleting in body tissues in general. However, signal redness is not simply a passive reflection of body carotenoid levels in this species. We have previously shown that concentrations of carotenoids in nuptial coloration far outweigh concentrations in the rest of the body, and moreover, low-carotenoid-diet males invest a much greater proportion of their total pool of carotenoids into ornaments (81\%) than do highcarotenoid-diet males (21\%; Pike et al. 2007). This strongly suggests that allocations of carotenoids to signals are under active control (and see Blount et al. 2003b).

The overall signaling dynamics at the population level are expected to depend on the condition distribution of the males at the beginning of the breeding season. As the initial condition of a male also affects his final fitness prospects, this might indicate strong selection for foraging ability or growth in the period leading up to the breeding season. Peuhkuri et al. (1995) showed experimentally in the three-spined stickleback that individuals in a group had a higher growth rate than did individuals reared on their own. They suggested that social facilitation in foraging and more effective predator avoidance might explain the result. In light of our findings, one might speculate that group living actually created the perception of a competitive environment in which it is important to grow fast and become relatively large. In the wild, selection for fast growth rates may also be balanced by selection in the opposite direction because fast growth is associated with reduced parasite resistance (Barber et al. 2001; Barber 2005).
Our model demonstrates how optimization of individual resource allocation in a competitive, game-theoretical setting can successfully predict the patterns in sexual signaling observed in an experiment with three-spined sticklebacks. However, we suggest that our approach is much more widely applicable to a broad range of species with sexual signaling, and so understanding how individuals optimize their allocation of resources to signaling in a competitive situation remains a major challenge for the future.

\section{Acknowledgments}

We thank Stirling University's Institute of Aquaculture for providing the antioxidant-free food in this study, G. Adam and J. Laurie for animal husbandry, and R. Reeve for discussions on the model. Two anonymous reviewers made excellent suggestions to improve the manuscript. This study was funded by a grant from the Natural Environment Research Council (to N.B.M, J.D.B, and J.L.). J.D.B. was supported by a Royal Society University Research Fellowship. This research adhered to the Association for the Study of Animal Behaviour Guidelines for the Use of Animals in Research and was performed under license from the UK Home Office.

\section{Literature Cited}

Alonso-Alvarez, C., S. Bertrand, G. Devevey, M. Gaillard, J. Prost, B. Faivre, and G. Sorci. 2004. An experimental test of the dosedependent effect of carotenoids and immune activation on sexual signals and antioxidant activity. American Naturalist 164:651-659.

Andersson, M. B. 1994. Sexual selection. Princeton University Press, Princeton, NJ.

Badyaev, A. V., and A. Qvarnström. 2002. Putting sexual traits into the context of an organism: a life-history perspective in studies of sexual selection. Auk 119:301-310.

Badyaev, A. V., and C. M. Vleck. 2007. Context-dependent development of sexual ornamentation: implications for a trade-off between current and future breeding efforts. Journal of Evolutionary Biology 20:1277-1287.

Barber, I. 2005. Parasites grow larger in faster growing fish hosts. International Journal for Parasitology 35:137-143.

Barber, I., S. A. Arnott, V. A. Braithwaite, J. Andrew, and F. A. Huntingford. 2001. Indirect fitness consequences of mate choice in sticklebacks: offspring of brighter males grow slowly but resist parasitic infections. Proceedings of the Royal Society B: Biological Sciences 268:71-76.

Blount, J. D., N. B. Metcalfe, T. R. Birkhead, and P. F. Surai. $2003 a$. Carotenoid modulation of immune function and sexual attractiveness in zebra finches. Science 300:125-127.

Blount, J. D., N. B. Metcalfe, K. E. Arnold, P. F. Surai, G. L. Devevey, and P. Monaghan. 2003b. Neonatal nutrition, adult antioxidant defences and sexual attractiveness in the zebra finch. Proceedings of the Royal Society B: Biological Sciences 270:1691-1696.

Candolin, U. 1999. The relationship between signal quality and physical condition: is sexual signaling honest in the three-spined stickleback? Animal Behaviour 58:1261-1267. 
2000a. Changes in expression and honesty of sexual signaling over the reproductive lifespan of sticklebacks. Proceedings of the Royal Society B: Biological Sciences 267:2425-2430.

2000b. Male-male competition ensures honest signaling of male parental ability in the three-spined stickleback (Gasterosteus aculeatus). Behavioral Ecology and Sociobiology 49:57-61.

Caswell, H. 2001. Matrix population models: construction, analysis and interpretation. Sinauer, Sunderland, MA.

Chaine, A. S., and B. E. Lyon. 2008. Adaptive plasticity in female mate choice dampens sexual selection on male ornaments in the lark bunting. Science 319:459-462.

Gautier, P., M. Barroca, S. Bertrand, C. Eraud, M. Gaillard, M. Hamman, S. Motreuil, G. Sorci, and B. Faivre. 2008. The presence of females modulates the expression of a carotenoid-based sexual signal. Behavioral Ecology and Sociobiology 62:1159-1166.

Hardouin, L. A., D. Reby, C. Bavoux, G. Burneleau, and V. Bretagnolle. 2007. Communication of male quality in owl hoots. American Naturalist 169:552-562.

Hill, G. E. 2006. Female mate choice for ornamental coloration. Pages 137-200 in G. E. Hill and K. J. McGraw, eds. Bird coloration. Harvard University Press, Cambridge, MA.

Höglund, J., and B. C. Sheldon. 1998. The cost of reproduction and sexual selection. Oikos 83:478-483.

Houston, A. I., and J. M. McNamara. 1987. Singing to attract a mate: a stochastic dynamic game. Journal of Theoretical Biology 129:57-68.

. 1999. Models of adaptive behaviour: an approach based on state. Cambridge University Press, Cambridge.

Hunt, J., R. Brooks, M. D. Jennions, M. J. Smith, C. L. Bentsen, and L. F. Bussière. 2004. High-quality male field crickets invest heavily in sexual display but die young. Nature 432:1024-1027.

Johnstone, R. A. 1995. Sexual selection, honest advertisement and the handicap principle: reviewing the evidence. Biological Reviews $70: 1-65$.

Johnstone, R. A., and A. Grafen. 1993. Dishonesty and the handicap principle. Animal Behaviour 46:759-764.

Judge, K. A., J. J. Ting, and D. T. Gwynne. 2008. Condition dependence of male life span and calling effort in a field cricket. Evolution 62:868-878.

Kokko, H. 1997. Evolutionarily stable strategies of age-dependent sexual advertisement. Behavioral Ecology and Sociobiology 41:99107.

Kolluru, G. R., G. F. Grether, S. H. South, E. Dunlop, A. Cardinali, L. Liu, and A. Carapiet. 2006. The effects of carotenoid and food availability on resistance to a naturally occurring parasite (Gyrodactylus turnbulli) in guppies (Poecilia reticulata). Biological Journal of the Linnean Society 89:301-309.

Lozano, G. A. 1994. Carotenoids, parasites, and sexual selection. Oikos 70:309-311.

Mappes, J., R. V. Alatalo, J. Kotiaho, and S. Parri. 1996. Viability costs of condition-dependent sexual male display in a drumming wolf spider. Proceedings of the Royal Society B: Biological Sciences 263:785-789.

McCauley, S. J., S. S. Bouchard, B. J. Farina, K. Isvaran, S. Quader, D. M. Wood, and C. M. St. Mary. 2000. Energetic dynamics and anuran breeding phenology: insights from a dynamic game. Behavioral Ecology 11:429-436.

McGraw, K. J., and D. R. Ardia. 2003. Carotenoids, immunocompetence, and the information content of sexual colors: an experimental test. American Naturalist 162:704-712.
McNamara, J. M., J. N. Webb, E. J. Collins, T. Székely, and A. I. Houston. 1997. A general technique for computing evolutionarily stable strategies based on errors in decision making. Journal of Theoretical Biology 189:211-225.

Milton, J. S., and J. C. Arnold. 1990. Introduction to probability and statistics. McGraw-Hill, New York.

Olson, V. A., and I. P. F. Owens. 1998. Costly sexual signals: are carotenoids rare, risky or required? Trends in Ecology \& Evolution 13:510-514.

Pérez-Rodríguez, L. 2008. Carotenoid-based ornamentation as a dynamic but consistent individual trait. Behavioral Ecology and Sociobiology 62:995-1005.

Peuhkuri, N., E. Ranta, S.-K. Juvonen, and K. Lindström. 1995. Schooling affects growth in the three-spined stickleback, Gasterosteus aculeatus. Journal of Fish Biology 46:221-226.

Pike, T. W., J. D. Blount, B. Bjerkeng, J. Lindström, and N. B. Metcalfe. 2007. Carotenoids, oxidative stress and female mating preferences for longer-lived males. Proceedings of the Royal Society B: Biological Sciences 274:1591-1596.

Proulx, S. R., T. Day, and L. Rowe. 2002. Older males signal more reliably. Proceedings of the Royal Society B: Biological Sciences 269:2291-2299.

Qvarnström, A. 2001. Context-dependent genetic benefits from mate choice. Trends in Ecology \& Evolution 16:5-7.

R Development Core Team. 2004. R: a language and environment for statistical computing. R Foundation for Statistical Computing, Vienna.

Sadd, B., L. Holman, H. Armitage, F. Lock, R. Marland, and M. T. Siva-Jothy. 2006. Modulation of sexual signaling by immune challenged male mealworm beetles (Tenebrio molitor, L.): evidence for terminal investment and dishonesty. Journal of Evolutionary Biology 19:321-325.

Sparkes, T. C., V. Rush, and S. A. Foster. 2008. Reproductive costs, condition, and carotenoid-based colour in natural populations of three-spine stickleback (Gasterosteus aculeatus). Ecology of Freshwater Fish 17:292-302.

Suk, H. Y., and J. C. Choe. 2008. Dynamic female preference for multiple signals in Rhinogobius brunneus. Behavioral Ecology and Sociobiology 62:945-951.

Torres, R., and A. Velando. 2003. A dynamic trait affects continuous pair assessment in the blue-footed booby, Sula nebouxii. Behavioral Ecology and Sociobiology 55:65-72.

von Schantz, T., S. Bensch, M. Grahn, D. Hasselquist, and H. Wittzell. 1999. Good genes, oxidative stress and condition-dependent sexual signals. Proceedings of the Royal Society B: Biological Sciences 266:1-12.

Welch, A. M. 2003. Genetic benefits of a female mating preference in gray tree frogs are context-dependent. Evolution 57:883-893.

Wilkinson, G., and G. E. Dodson. 1997. Function and evolution of antlers and eye stalks in flies. Pages 310-328 in J. C. Choe and B. J. Crespi, eds. The evolution of mating systems in insects and arachnids. Cambridge University Press, Cambridge.

Wong, B. B. M., and A. P. Svensson. 2009. Strategic male signaling effort in a desert-dwelling fish. Behavioral Ecology and Sociobiology 63:543-549.

Wootton, R. J. 1976. The biology of the sticklebacks. Academic Press, London.

Associate Editor: Anna Qvarnström Editor: Michael C. Whitlock 